\title{
Topical polidocanol application in combination with static stretching in tendinopathies: a prospective pilot study
}

\author{
Matthias Gatz 1 \\ Simone Schrading ${ }^{2}$ \\ Timm Dirrichs ${ }^{2}$ \\ Marcel Betsch ${ }^{1}$ \\ Markus Tingart ${ }^{1}$ \\ Björn Rath ${ }^{1}$ \\ Christiane Kuhl ${ }^{2}$ \\ Valentin Quack ${ }^{1}$ \\ 1 Department of Orthopaedics, RWTH Aachen, \\ Germany \\ 2 Department of Diagnostic and Interventional Radiol- \\ ogy, RWTH Aachen University Hospital, Germany
}

Corresponding author:

Matthias Gatz

Department of Orthopaedics, RWTH Aachen

Pauwelsstraße 30

52074 Aachen, Germany

E-mail: Matthias-Gatz@gmx.de

\section{Summary}

Background: Highly operator-based injection therapy with vasosclerosing and anesthetic polidocanol is used for tendinopathies. This pilot-study evaluates the topical application of polidocanol gel.

Methods: Prospective case series. 39 patients with tendinopathies (14 Achilles, 14 patella, 11 wrist extensors) with a symptom duration $>6$ months were included. Polidocanol and static stretching were the therapy in the first 2 weeks, while static stretching was continuously performed for 6 months. Clinical outcome was evaluated with VISA-A, VISA-P and DASH Scores and sonographically with B-Mode (B-Mode), Power Doppler (PD) and Shearwave Elastography (SWE). Results: 22 patients clinically improved $(>+10$ score points), 11 patients were without improvement $(<+10)$ and 1 patient worsened during 6 months. The VISA-A Score increased in average 19 points from 56 to $75(p<0.01)$, VISA-P Score increased 13 points from 59 to $72(p<0,01)$ and the DASH-Score decreased 20 points from 40 to 20 $(p<0,01)$. SWE correlates better than B-Mode or PD with symptom improvement.

Conclusion: The combination of static training and topical polidocanol application seems to be successful in the treatment of tendinopathies. Further RCT studies need to evaluate the efficiency of topical polidocanol application. SWE is a more sensitive tool to describe symptom development than PD or B-Mode.

Level of evidence: IV.

KEY WORDS: Achilles, epicondylitis, patella, shear wave elastography, tendinopathy, ultrasound.

\section{Introduction}

Most common tendinopathies (TP) are at the Achilles, patella and wrist extensor tendons.

\section{Neovascularisation as a pathogenic model in chronic TPs}

The pathogenesis of chronic TPs is not fully understood, but it is based on overuse combined with various individual risk factors resulting in both disturbance of tendon extracellular matrix levels and decrease of tenocyctes cell numbers ${ }^{1,2}$. A posttraumatic inflammatory response is present with leucocytes suppressing in vitro Type 1 collagen synthesis, which might be a stimulus for catabolic and pro-inflammatory mediators ${ }^{3}$. According to the Iceberg Theory of Abate relative overload leads to micro ruptures, followed by the over-expression of Vascular Epithelial Growth Factor (VEGF) ${ }^{4}$. As a result, VEGF stimulates the synthesis of matrix metalloproteinases (MMPs) with a imbalance of MMPs and tissue inhibitors of MMPS, leading to degradation of the extracellular matrix consecutively decreased collagen strength and a predisposition of further micro ruptures ${ }^{2,5}$. Secondly, VEGF induces neovascularization (neoV) covered by nerve fibers, which can be activated by local cytokines as well as by cholinergic and sympathetic innervation of these peripheral nerves ${ }^{6}$. Even if nerve supply differs by tendon region, in a painful tendon free nerve endings are remarkable high concentrat$\mathrm{ed}^{2}$. In symptomatic tendons neoV is a common finding in Power Doppler Ultrasound with corresponding angiogenesis in histological preparations, but "it is difficult to know if this neoV represents genuine

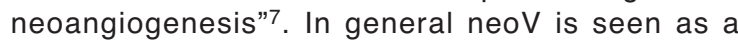
pathological vascular ingrowth in the symptomatic tendon, but there also exists opinion that the pain and the whole pathogenetic model are not totally based on neo ${ }^{8}$. Therefore, in addition to the neurovascular ingrowth further aspects like synthesis rates of gluta- 
mate, substance $\mathrm{P}$ and calcitonin gene related peptide also play a role in TP. Increased levels of theses substances provoke a neurogenic-mediated inflammation. Working together, both neurogenic-mediated inflammation and neoV are seen as an inappropriate healing process with neoV being a result of the inflammatory response ${ }^{7}$. At this point pain just gets present. As a result, this pilot study concentrates on a therapeutical approach to reduce neoV and the accompanied nerve fibers in order to reduce pain and break through the cascade of tendinopathy ${ }^{4}$.

\section{Invasive and non-invasive treatments}

Conservative treatment options of TP are eccentric exercises, extracorporeal shock wave therapies or injection therapies with high-volume injections, plateletrich plasma, corticosteroids or polidocanol ${ }^{9}$. A Cochrane Review evaluating RCT studies of various injection therapies in AT draw the conclusion that there is no sufficient evidence for a routine injective use $^{9,10}$. Consequently, non-invasive topical applications could be a further option as an independent home-based standard medication to the already established NSAIDS or nitroglycerine ${ }^{11}$. In general, there is a high interest and acceptance in patients for a topical rather than a systemic medication, ease of use and an attempt for an alternative to NSAIDs. In that sense, a natural based oxygen oil with an unknown active component showed significant effects in acute tendinopathies compared to a placebo ${ }^{12}$. Concerning conventional active pharmaceutical ingredients, Frizziero et al. examined a betamethasone plaster in elbow tendinopathy significantly reducing the VAS score by twice with a 12 hour application compared to a placebo ${ }^{13}$. Furthermore, there is a growing interest of alternative options like nutraceutical supplements e.g. curcumin, which inhibits neoV and apoptosis in addition to an antioxidant effect showing good results in animal models ${ }^{14}$.

\section{Polidocanol as a causal treatment option}

Polidocanol is a sclerosing, antipruritic and anesthetic agent. Injected, it is mainly used for sclerosing varices in the lower extremities, while in psoriasis it is cutaneously applied ${ }^{15}$. In solution polidocanol molecules are mainly enclosed in a micelle, while free molecules are surface-active and attach to the endothelial wall causing a disintegration and denaturation; thus thrombocytes get adherent causing a sclerosing effect ${ }^{16}$. Antipruritic effects could be explained by inhibiting unmyelinated C-nerve fibers. Polidocanol should induce ischemia to reduce the neural coat of the vessels and to break through the pathologic healing process ${ }^{17}$. Alfredson and Öhberg introduced an injection technique for Achilles TP, which was subsequently used in patella and wrist extensors tendons ${ }^{18,19}$. Using Power Doppler sonography polidocanol should be injected intravascularly directly into the neoV. Nevertheless, extravascular injection is also effective, most likely by ischemic effects on nerve fibers ${ }^{17}$. The results of the injection technique were good. In patella TP the VISA- Score increased of 26 points during 1 year ${ }^{20}$. In the Achilles tendon VAS score reduces from 75 to $7^{21}$ and in wrist extensors from 75 to $34^{22}$. Whereas, in a further study by van Sterkenburg $50 \%$ of Achilles TP patients had a poor outcome, which might be explained by the retrospective study design without consistent use of VISA-A Scores ${ }^{23}$. However, as Alfredson has written "the method is technically demanding, very much operator-dependent with a long learning curve, making it less suitable for general use" 24 . Therefore this pilotstudy investigates the topical application of polidocanol in combination with static stretching to avoid operator-based disadvantages and to provide a general, non-invasive form of topical application without technical devices. In contrast to injection therapy where the active agent is directly applied to the area of interest the quantitative absorption of the skin needs to be considered. Concerning Polidocanol or related substances, just $2 \%$ of the topically applied amount penetrates the human skin ${ }^{15,25}$. Furthermore, the absorption rate of the ampiphil polidocanol through the Stratum corneum of the skin might be enhanced by allantoin, which is as an ingredient of the product (Thesit $\left.{ }^{\circledR}\right)$ used in this study and in general meliorates skin penetration. Unfortunately, no data exist which scientifically quantify a possible increase of the $2 \%$ absorption rate by different galenic mechanisms or allantoin. It is our understanding that polidocanol has to penetrate the skin without any support by cutaneous transport carriers.

\section{Materials and methods}

\section{Study design}

We chose a prospective case series study as a study design. The investigation was approved by the ethical committee of our medical faculty (EK 267/12) and meets the ethical standards of the Declaration of Helsinki and the MLT Journal ${ }^{26}$. All volunteers provided written informed consent to participate.

\section{Inclusion and exclusion criteria}

In total, 36 patients were included: 14 patients with Achilles, 11 with patella and 11 with wrist extensors TP (Table I). All patients experienced pain or discomfort while participating in their sports or at work.

They suffered from chronic TP of the patella, Achilles or wrist extensor tendons for at least 6 months and were pre-diagnosed and treated individually non-successfully by physicians previously with various therapies. Diagnosis was confirmed by clinical history and tests, pain questionnaire and sonographic examination.

To focus on severe tendinopathies, 9 eligible patients with low symptomatic (>80 VISA-A/P; <20 DASH) were excluded. Other exclusion criteria were inability to perform stretching exercises, local cortisone injection in the last 6 months, previous surgery or rupture of the tendon, rheumatic diseases and medication with fluoroquinolone. 
Table I. Patient characteristics in entire cohort.

\begin{tabular}{|c|c|c|c|c|c|c|}
\hline \multirow{2}{*}{$\begin{array}{l}\text { Characteristics } \\
\text { Sex }\end{array}$} & \multicolumn{2}{|c|}{ Achilles $(n=14)$} & \multicolumn{2}{|c|}{ Patella $(n=11)$} & \multicolumn{2}{|c|}{ Wrist extensor $(n=11)$} \\
\hline & 우 5 & $\sigma^{7} 9$ & 우 0 & o 11 & 우 6 & 万 5 \\
\hline Age & \multicolumn{2}{|c|}{$50 \pm 13(31-69)$} & \multicolumn{2}{|c|}{$26 \pm 5(20-40)$} & \multicolumn{2}{|c|}{$49 \pm 15(24-70)$} \\
\hline \multicolumn{7}{|c|}{ Side of affected tendon } \\
\hline Left & \multicolumn{2}{|l|}{10} & \multicolumn{2}{|l|}{7} & \multicolumn{2}{|l|}{3} \\
\hline Right & \multicolumn{2}{|l|}{8} & \multicolumn{2}{|l|}{7} & \multicolumn{2}{|l|}{9} \\
\hline \multicolumn{7}{|c|}{ Symptom duration in months } \\
\hline Mean & \multicolumn{2}{|l|}{24} & \multicolumn{2}{|l|}{30} & \multicolumn{2}{|l|}{20} \\
\hline Median & \multicolumn{2}{|l|}{12} & \multicolumn{2}{|l|}{11} & \multicolumn{2}{|l|}{9} \\
\hline Min.- max. & \multicolumn{2}{|c|}{$6-84$} & \multicolumn{2}{|c|}{$6-102$} & \multicolumn{2}{|c|}{$24-70$} \\
\hline
\end{tabular}

Previous therapeutic approaches

\begin{tabular}{l|l|l|l}
\hline Mean & 3,86 & 3,75 & 4 \\
\hline Cryotherapy & 9 & 11 & 5 \\
\hline NSAID & 8 & 11 & 8 \\
\hline Cortisone & 1 & 2 & 2 \\
\hline $\begin{array}{l}\text { Professional } \\
\text { physiotherapy }\end{array}$ & 10 & 11 & 7 \\
\hline Shock wave & 3 & 0 & 3 \\
\hline Acupuncture & 3 & 1 & 1 \\
\hline Bandage & 2 & 11 & 11 \\
\hline
\end{tabular}

Activity-loading

\begin{tabular}{l|l|l|l}
\hline Mean hours/week & 4,5 & 8 & 1,68 \\
\hline Recreational sport & 11 & 1 & 3 \\
\hline Amateur sport & 0 & 10 & 0 \\
\hline Work related & 0 & 0 & 4 \\
\hline Level of pain & 2 & 5 & 4 \\
\hline In rest & 11 & 10 & 9 \\
\hline During palpation & 10 & 13 & 9 \\
\hline During activity & 9 & 12 & 8 \\
\hline After activity & 9 & \multicolumn{1}{|l}{} \\
\hline
\end{tabular}

\section{Study design}

There were 3 scheduled assessment visits: at the beginning ( 0 month), after 1 and 6 months. At every visit patients were examined clinically by two independent physicians (Flowchart I).

The therapy consisted of two elements: static stretching exercises and cutaneous polidocanol application. In the first two weeks patients used polidocanol gel, 


\section{Entire Cohort:}

Information with written informed consent

48 patients in total

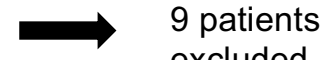

- 19 Achilles

excluded

- 16 Patella

- 13 wrist extensor

39 patients

included

\section{Date 1:}

Clinical and sonographical evaluation

39 patients in total

- 14 Achilles

- 14 Patella

- 11 wrist extensor

Stretching and

cutaneous

Polidocanol

\section{Date 2 (after 1 month):}

Clinical and sonographical evaluation

36 patients in total

- 14 Achilles

- 11 Patella

- 11 wrist extensor

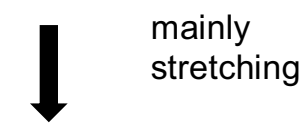

\section{Date 3 (after 6 month):}

Clinical and sonographical evaluation

34 patients in total

- 12 Achilles

- 11 Patella

- 11 wrist extensor

$\Longrightarrow 3$ patients lost due

to non-compliance

Flowchart I: Study design. 
performed stretching exercises and did not participate in sports. After two weeks patients could individually slowly start loading and continue a load level depending upon pain (scale 4/10) and discomfort until their full recovery.

We used the commercially available Thesit ${ }^{\circledR}$ gel $50 \mathrm{~g}$ (Gepepharm GmbH, Hennef Germany) with the standard concentration of $8 \%$ for topical application. Patients were instructed to apply an amount as large as a cherry pit of Thesit ${ }^{\circledR}$ gel 3 times a day, topically for the first 2 weeks. There were no reported side effects.

At the first appointment patients were taught how to perform static stretching exercises at home. They were instructed to perform the same at least once (up to 3 times) per day and to hold the position for 30 seconds. Additionally, they received a document presenting these exercises. For Achilles and patella tendon both groups stretched statically hip flexors/extensors, knee flexors/extensors and the plantar flexors (M. triceps surae) in 5 different exercises. For wrist extensor TP patients stretched the extensors carpi radialis and the flexor carpi ulnaris in 2 different exercises. At the second visit we reviewed the correct performance of stretching and evaluated stretching compliance by asking, how many times per day or week exercises have been performed. Stretching was continued during the whole study. We chose static stretching over eccentric exercises (EE), since static stretching is easier to apply without having to use additional devices such as decline boards or rubber bars. In addition, there are studies showing no benefits of EE over static exercises in AT or wrist extensor $T P$, e.g. Frizziero et al. reported $a^{27-29}$ superiority of EE in only $2 / 9$ studies in wrist extensor TP. For a better comparison we used static stretching for all the 3 different types of TP in our study.

\section{Clinical evaluation by VISA-A/P and DASH}

For pain evaluation (0/1/6 month) the validated Victorian Institute of Sports Assessment (VISA) questionnaires in German for Achilles (VISA-A) and patella TP (VISA-P) had been chosen ${ }^{30,31}$. Each consists of 10 differently weighted questions with a total of 0 (highest pain) to 100 points (no pain at all). For evaluation of wrist extensor TP we chose the German version of the Disability Arm Shoulder Hand (DASH) Score ${ }^{32}$ which consists of 30 equally weighted questions with a total of 100 (highest pain) to 0 points (without pain). Questionnaires were filled in 0,1 and 6 months. Relevant improvement or worsening was decided by a minimum change of $+10 /-10$ score points. Scores over 90 (VISA) or 10 (DASH) were considered as excellent outcome ${ }^{33}$.

\section{Sonographic evaluation}

Sonographic evaluation was done by B-mode ultrasound (US), power-Doppler sonography (PD) and Shear Wave Elastography (SWE) with a high-resolution linear $15 \mathrm{MHz}$ transducer (AixplorerTM, SuperLinearTM SL 15-4, Supersoniclmagine, Aix-enProvence France) with a band-width of 4-15 MHz. In every participant a gel cushion delay block (Sonogel, Sonokit Proxon, length $100 \times 100 \mathrm{~mm}$, delay distance $20 \mathrm{~mm}$ ) was used for better docking. Two experienced radiologists with at least 5 years of experience in musculoskeletal sonographic imaging performed all sonographic examinations. For comparison both tendon sides were evaluated without knowledge of the affected side. Tendons were examined in relaxed stance: the examination of Achilles tendon was done in prone position, the patient's foot project beyond the examination table and in neutral subtalar position. The examination of patellar tendon was done with the patient in dorsal position, the leg in relaxed extended position. Wrist extensor tendons got examined in a sitting position with the forearm lying on the tigh in a slightly flexed position. The acquired SWE-information was evaluated quantitatively in Kilopascal ( $\mathrm{kPa}$ ) up to a maximum tissue rigidity of $300 \mathrm{kPa}$ at three representative locations in the proximal, mid and distal part of the tendon. Mean (SWE mean) and maximum (SWE max) tissue rigidity was quantitatively evaluated by ROI-based analysis in the most rigid area in every image. Standardized diameter of the ROI was $3 \mathrm{~mm}$. NeoV was graduated by the Öhberg score $(0-4)^{18}$. Morphological changes (B-Mode) in the tendons were classified by $0=$ none, to degree $4=$ major change.

\section{Statistical analyses}

For all analyses, SPSS 22.0 was used to assess statistical significance, which was defined as $p<0.05$. The T-Test with connected variables compares the groups before and after treatment. Furthermore, a one-way ANOVA with repeated measures (Greenhouse-Geisser correction) were made for each tendon localisation to examine significant changes over time. Post hoc tests used the Bonferoni correction. Spearman's correlation coefficient analyses correlation between the VISA-A/P; DASH score and the sonographic parameters as B-Mode, Öhberg Score and SWE. By this we compared the difference between the first and the last score after 6 months with the difference in the sonographic parameters during this time.

\section{Results}

\section{General results}

In summary, 22 patients improved, 11 patients did not feel a relevant improvement, while 1 patient worsened. 9 patients reached scores over 90 points (VISA-A/P) or less than 10 points (DASH). Furthermore, 6 patients reached points over 80 points (VISA$\mathrm{A} / \mathrm{P}$ ) / under 20 points (DASH), showing encouraging results in nearly half of the patients. All patients used the total $50 \mathrm{~g}$ Thesit ${ }^{\circledR}$ gel tube during the 6 months. As Thesit ${ }^{\circledR}$ is free for sale, 5 of the 11 patients without improvement after one month informed us about using additional $50 \mathrm{~g}$, but still they did not improve more than 10 points in total. Compliance for stretching exercises and gel application was good. In the 
first month, all participants were performing the exercises at least once per day, while in the following period of 5 months monitoring of compliance was difficult, but participants were able to state a performance of at least 4 times per week. There were no reported side effects of therapy. No correlation of outcome relating to type of loading-activity, gender, higher or lower initial score could be found.

\section{Achilles tendon}

For Achilles TP $(n=14)$ the mean initial VISA-A score was 56 (range 26-68, SD 15) and increased to 65 (range 35-85, SD 13) after 1 month and to 75 (range 10-97, SD 25) after 6 months (Diagram I). Initial scores on average rose about 19 points (range -16 to 53 , SD 21) during 6 months and increased significantly from the beginning $(p<0,01)$. The repeated ANOVA with a Greenhouse-Geisser correction determined that mean VISA-A differed statistically significantly between the measure points $[F(1,1155 ; 12,700)$ $=7,859, p<0,013]$. Post hoc tests using the Bonferoni correction revealed that there were significant changes between the beginning and after the 1 . month $(p=0,005)$ as well as after 6 months $(p=0,02)$, while after the 1 . until the 6 . month there was no significant change $(p=0,229)$. During therapy 1 patient worsened, 3 patients showed no improvement and 8 patients improved. Half of these 8 patients $(n=4$, mean initial score: 57 ) reached a mean final score of 95. Symptom improvement during 6 month correlated moderately with SWE mean $(r=0,46 ; p=0,16)$ and $\max (r=0,48 ; p=0,14)$ values, while B-Mode $(r=0)$ and PD $(r=0,13)$ did not correlate at all.

\section{Patella tendon}

Initial mean VISA-P score for patella TP $(n=11)$ was 59 (range 43-78; SD \pm 11 ), increased to 67 points (range 46-87; SD \pm 13 ) after 1 month and rose up to 72 (range 53-98; SD \pm 15 ) at 6 months (Diagram II). Initial scores on average rose about 13 points ( -3 to 43; SD \pm 14 ) during 6 months and increased significantly from the beginning $(p<0,01)$. ANOVA yield that there is a significantly change between time points $[F(1,954 ; 19,536)=5,596, p<0,012]$ with a significant score difference between the beginning and after 6 months $(p=0,025)$ in the post hoc tests. Between the beginning and the 1 . month $(p=0,175)$ and the 1 . month until the 6 . months $(p=0,686)$ there were no significant changes. During therapy no patient worsened, 5 patients maintained the level of pain, while 6 improved with 1 reaching a score of 98. SWE mean $(r=0,6 ; p=0,05)$ and SWE max $(r=0,42 ; p=0,20)$ changes correlated moderately with symptom-alteration during the 6 month follow-up, in contrast to B-Mode $(r=0)$ and PD $(r=-0,5)$, which did not correlate at all.

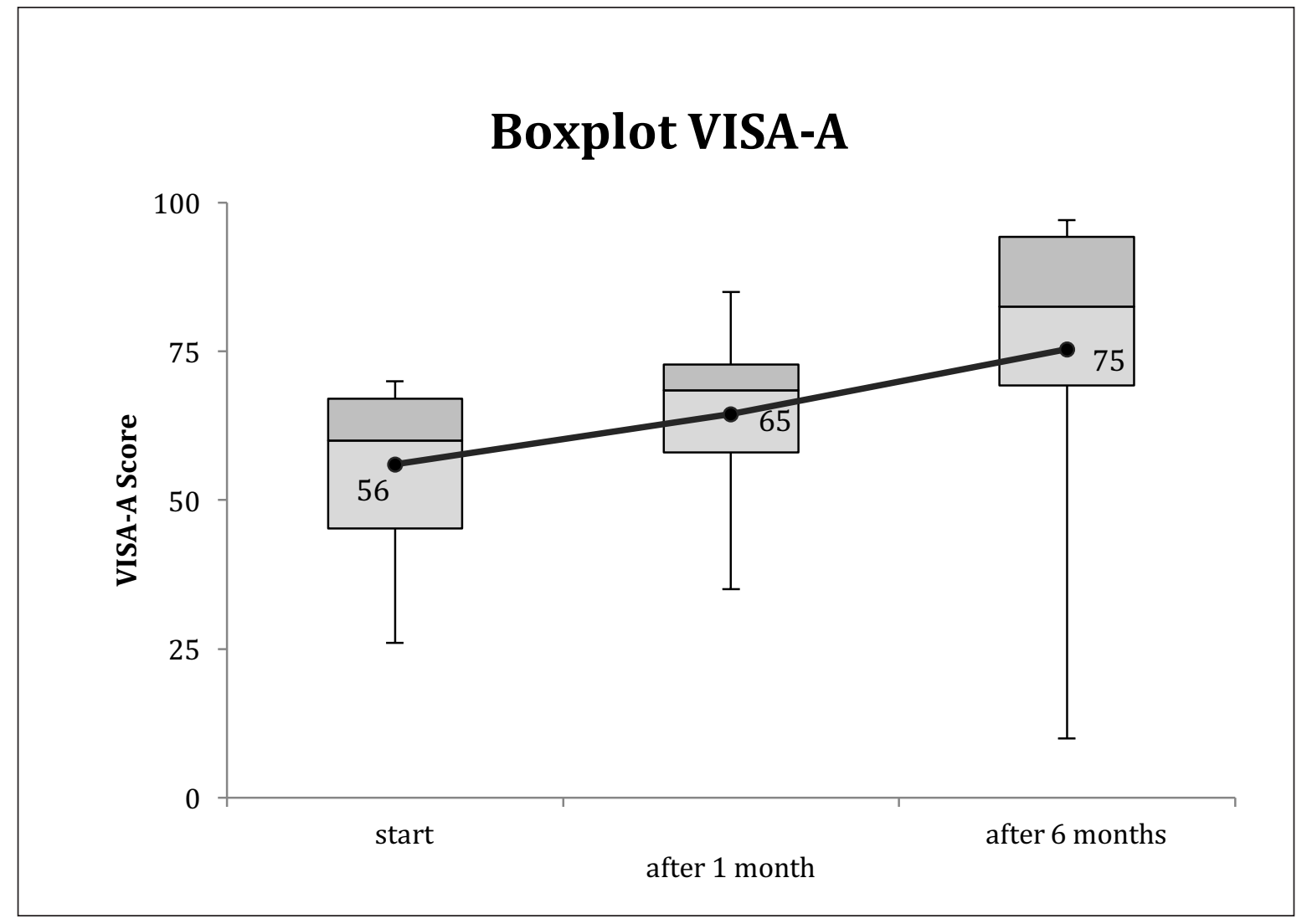

Diagram I. Progression in Achilles tendinopathy. 


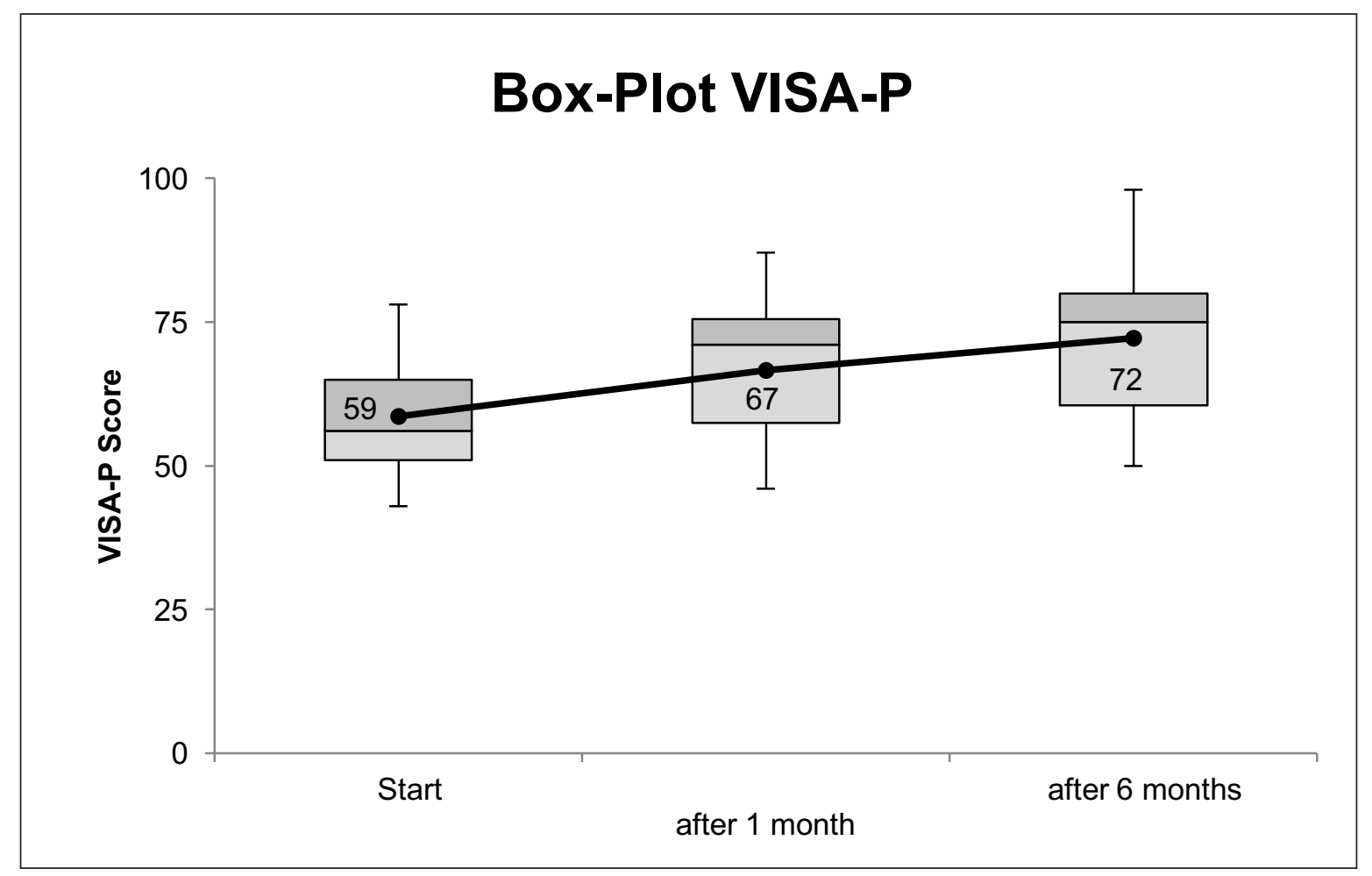

Diagram II. Progression in patella tendinopathy.

\section{Wrist extensor}

DASH- Score for wrist extensor TP $(n=11)$ decreased from initially 40 points (range $49-25$; $S D \pm 7$ ) to 28 (range 56-5; SD \pm 15 ) after 1 month and reached 20 points (range 50-2; SD \pm 15 ) at the end of 6 months (Diagram III). Initial scores decreased 20 points (range $-38-6, S D \pm 14$ ) in total during 6 months and differ significantly from the beginning $(p<0,01)$. ANOVA showed a statistically significant difference between the measure points $[F(1,934 ; 19,337)=13,098$, $\mathrm{p}<0,0005]$. There are significant changes in the post hoc tests between the beginning and after the 1 . month $(p=0,038)$ and 6 month $(p=0,02)$ respectively, while after the 1 . until the 6 month $(p=0,164) \mathrm{DASH}$ increase was not significant. During therapy no patient worsened significantly, 3 patients stayed nearly at the same level, while 8 improved with 4 healed patients reaching scores under 7,5. During the whole period of follow-up, SWE mean $(r=0,54 ; p=0,09)$ and SWE max $(r=0,47 ; p=0,14)$ changes correlate moderately with symptom-alteration, in contrast to B-Mode $(r=0)$ and to PD $(r=-0,5)$, which remained nearly constant.

\section{Discussion}

This pilot study investigates the topical application of polidocanol combined with static stretching exercises in three different types of chronic TPs (patella,
Achilles, wrist extensors) showing good to excellent clinical results in nearly half of the patients. There is no correlation with previous activity loading, symptom duration or gender, making it suitable for all kinds of patients suffering from TPs. In Achilles tendons and wrist extensors the outcome was similar $(+19 / 20$ points), while in patella TP the average improvement was 13 points. First, it is difficult to compare different scores despite the same range, as a specific change in one score is not valid the same change in the others. Secondly, neoV in patella tendons coming from the deep infra-patellar fat pad might be insufficiently reachable by topical application ${ }^{34}$. Although, neoV in the Achilles tendon seems to come from the ventral side of the tendon as well, it might be better reachable through lateral application ${ }^{18}$.

Making general statements about the natural course of healing is difficult and patients differ a lot e.g. in risk factors or loading. Symptoms of patella TP in amateur athletes last in average 18 months $^{35}$. In Achilles TP $29 \%$ fail conservative treatment ${ }^{36}$. Wrist extensor TP seems to be self-limiting during the first 8-13 months ${ }^{37}$. To sum up, normal healing process during 6 months could only play a minor role in explaining our results.

Comparison to other therapeutic modalities is difficult due to different scoring systems, different activity loadings and symptom intensity. In our study on average patients' VISA-A increased about 19 points during 6 months. In a randomized controlled clinical 


\section{Boxplot DASH}

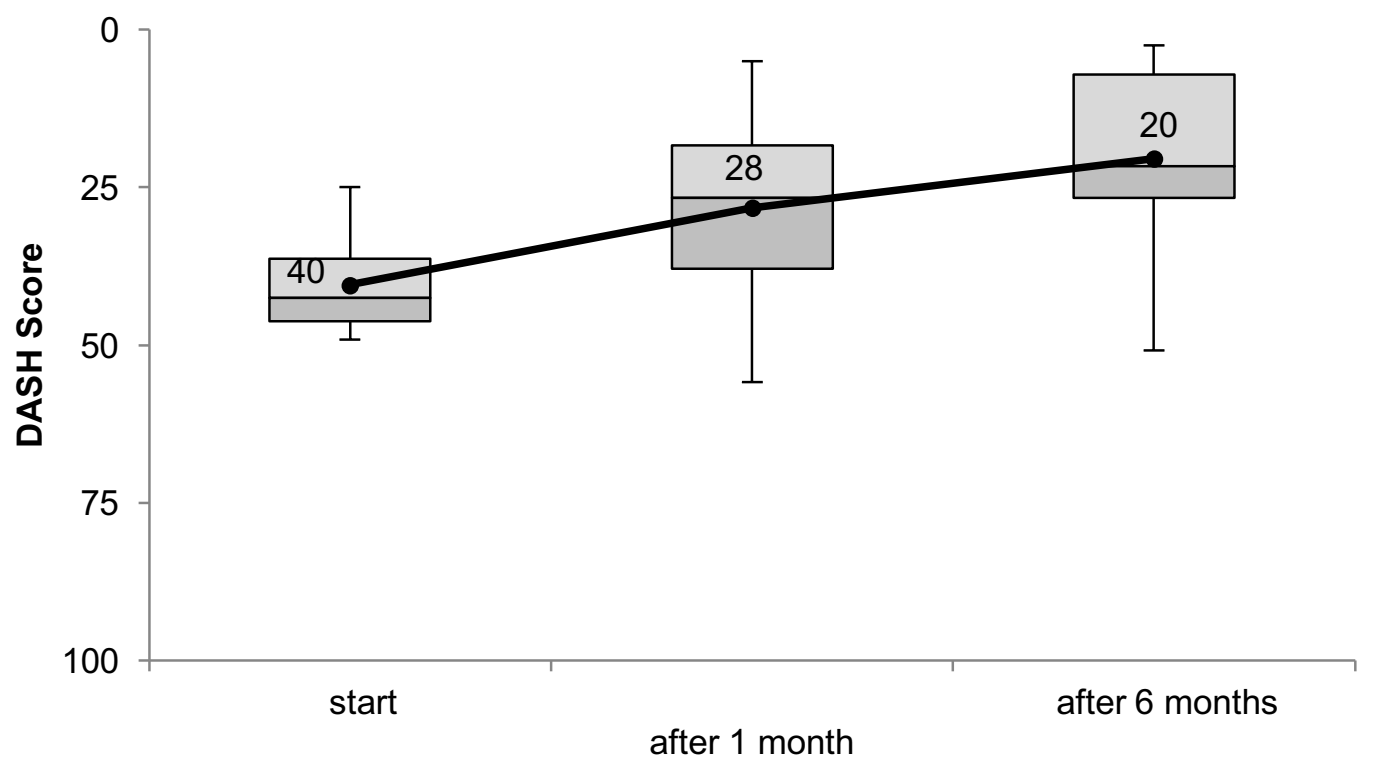

Diagram III. Progression in wrist extensor tendinopathy.

trail glyceroltrinitrat showed good results: twentyeight $(78 \%)$ of thirty-six Achilles tendons in the glyceryl trinitrate group were asymptomatic with activities of daily living at 6 months, but some patients had reversible side effects like headache and rash $^{38}$. VISA-Score was just used in one study about polidocanol injection therapy. In it, VISA-P points increased about 26 after one year 20 , while we reached 13 points with the topical application after half a year.

A drawback of our study is that it is unclear, if polidocanol reached the area of interest after penetrating the skin due to a lag of pharmakokinetic and dynamic datas regarding topically applied polidocanol. However, at least $2 \%$ of cutaneous applied polidocanol or related substances are absorbed through the human skin $^{15,25}$. Using a $50 \mathrm{~g}$ tube polidocanol gel $(50.000$ $\mathrm{mg}$ ) with a concentration of $8 \%$ and a minimal absorption rate of $2 \%$, theoretically at least $80 \mathrm{mg}$ $\left(50.000 \mathrm{mg}^{\star} 0,08^{\star} 0,02\right)$ should penetrate the skin and might reach the area of interest. This would be equal to $16 \mathrm{ml}$ of injected polidocanol (standard dosage 5 $\mathrm{mg} / \mathrm{ml}$ ) or 8 intravasal injections (2 ml per injection) ${ }^{17}$. Furthermore, evaluation of the polidocanol's effect can be solely made by clinical improvement, as neoV remained mainly constant and did not correlate with symptom improvement. Nonetheless, if a total sclerosis is necessary for symptom improvement is questionable. Directly after polidocanol injections no blood flow is noticeable, followed by a higher blood flow af- ter 1-3 weeks ${ }^{39}$. It seems that neoV in some cases is not pain related and not adequate for evaluating the therapeutic effect for injected and topical polidocanol. Referring to the pathogenesis model mentioned in the introduction polidocanol is able to address neoV and its accompanying nerves, but not the posttraumatic inflammatory response of leukocyctes or the effects of MMPs induced by VEGF. Additionally, Willberg assumed ischemic effects on nerve fibers accompanying neoV in TP (17), which might explain the longterm symptom improvement despite the recurrent neoV. Besides the sclerosing effect of polidocanol, an additional anesthetic effect is often quoted, but nowadays discussed controversially regarding topical application. Mainly older studies state an anesthetic potential by injective treatment, while topically applied polidocanol was not showing any anesthetic potential in thermal sensory analysis and pinpricking 40,41 . The exact effect of injected and topical applied polidocanol concerning TP is still unexplored, nevertheless in 2005 a RCT study reported a positive effect of polidocanol on symptom improvement in TP42. Moreover, in a special formula with a lipophilic base polidocanol concentration can be increased up to $9 \%$ and potentially even more of the agent could reach the area of interest. In contrast, in injection therapy there is no significant evidence that higher dosage $(5 \mathrm{mg} / \mathrm{ml}$ up to $10 \mathrm{mg} / \mathrm{ml}$ ) improves the outcome ${ }^{17}$. It would be of interest in future studies to examine higher dose cutaneous application in a RCT trial. 
Static exercises could have an influence on the final outcome, but studies alone using VISA-A/-P or DASH scores were not found on pubmed or PEDro. Stasinopoulos reported excellent results after one month of a combination of static stretching and eccentric exercises $(+42$ VISA-P) in patients with patella TP while eccentric exercises alone reached +28 points. In contrast to Stasinopoulos' patients, ours had a higher grade of severity, more therapeutical approaches (3,75 compared to 1$)$ and longer mean symptom duration (12 months compared to 6$)^{43}$. Nevertheless, eccentric home based exercises can also lead to an unsatisfactory increase of 11 VISA-A after 6 months $^{44}$.

To sum up, in this conservative approach both the physical exercises and the polidocanol application can be responsible for symptom improvement without knowing the exact influence of each part. ANOVA revealed in wrist extensors and Achilles TP a significant change in the first month, where polidocanol was used daily in the first 2 weeks. In the period where mainly stretching exercises were used, there was no significant score difference, giving a hint that daily usage of Thesit@ has a further effect than stretching exercises alone.

SWE seems to display tendon healing better than US or PD and might be a useful tool for follow-up of tendinopathies under therapy. Conventional ultrasound provides "findings on imaging creating a confusing clinical picture" with a questionable possibility of monitoring ${ }^{45}$. However in this study correlation of SWE with clinical scores and symptom alteration was better than standard sonographic B-Mode or Power Doppler showing that SWE might be a powerful tool for monitoring. Further studies investigating the correlation between score changes and imaging changes of different treatment options need to evaluate the potential of SWE. Even though SWE is a relatively new tool in tendon diagnostics, it is already known that asymptomatic tendons are harder than symptomatic ones ${ }^{46}$. In conclusion, increased tendon rigidity during the 6 month follow-up of our study, refutes a single placebo effect of the combined polidocanol and stretching therapy.

In summary, $45 \%$ of the patients suffering from chronic TP reached good or excellent clinical results. Further blinded randomized prospective studies need to evaluate the efficiency of polidocanol in comparison to currently used treatment options.

\section{Conflict of interest}

The Author has no financial or personal relationships with other people or organizations that could inappropriately influence their work.

\section{References}

1. Freedman BR, Gordon JA, Soslowsky LJ. The Achilles tendon: fundamental properties and mechanisms governing heal- ing. Muscles Ligaments Tendons J. 2014;4(2):245-255.

2. Reinking M. Tendinopathy in athletes. Phys Ther Sport. 2012;13(1):3-10.

3. Al-Sadi O, Schulze-Tanzil G, Kohl B, Lohan A, Lemke M, Ertel $\mathrm{W}$, et al. Tenocytes, pro-inflammatory cytokines and leukocytes: a relationship? Muscles Ligaments Tendons J. 2011; 1(3):68-76.

4. Abate M, Silbernagel KG, Siljeholm C, et al. Pathogenesis of tendinopathies: inflammation or degeneration? Arthritis Res Ther. 2009;11(3):235.

5. Del Buono A, Oliva F, Osti L, Maffulli N. Metalloproteases and tendinopathy. Muscles Ligaments Tendons J. 2013;3(1):5157.

6. Danielson P. Reviving the "biochemical" hypothesis for tendinopathy: new findings suggest the involvement of locally produced signal substances. Br J Sports Med. 2009;43(4):265268.

7. Rees JD, Stride M, Scott A. Tendons-time to revisit inflammation. Br J Sports Med. 2014;48(21):1553-1557.

8. Tol JL, Spiezia F, Maffulli N. Neovascularization in Achilles tendinopathy: have we been chasing a red herring? Knee Surg Sports Traumatol Arthrosc. 2012;20(10):1891-1894.

9. Loppini M, Maffulli N. Conservative management of tendinopathy: an evidence-based approach. Muscles Ligaments Tendons J. 2011;1(4):134-137.

10. Kearney RS, Parsons N, Metcalfe D, Costa ML. Injection therapies for Achilles tendinopathy. Cochrane Database Syst Rev. 2015(5):CD010960.

11. Diehl P, Gollwitzer H, Schauwecker J, Tischer T, Gerdesmeyer L. [Conservative treatment of chronic tendinopathies]. Orthopade. 2014;43(2):183-193.

12. Pappagallo M, Leslie JB, Raffa RB, Kash P, Fleischer C, Sinclair $\mathrm{N}$, et al. Evaluation of a novel topical essential oxygen oil for the treatment of pain in acute tendinopathy and sprains. Muscles Ligaments Tendons J. 2011;1(1):25-30.

13. Frizziero A, Causero A, Bernasconi S, Papalia R, Longo M, Sessa $V$, et al. Efficacy of betamethasone valerate medicated plaster on painful chronic elbow tendinopathy: a double-blind, randomized, placebo-controlled trial. Muscles Ligaments Tendons J. 2016;6(1):131-139.

14. Fusini F, Bisicchia S, Bottegoni C, Gigante A, Zanchini F, Busilacchi A. Nutraceutical supplement in the management of tendinopathies: a systematic review. Muscles Ligaments Tendons J. 2016;6(1):48-57.

15. (BfR) BfR. Polidocanol in kosmetischen Mitteln. Stellungnahme des BfR vom 15. 2003.

16. Kreussler CF. Der Wirkstoff - Wie wirkt Aethoxysklerol? 2012.

17. Willberg L, Sunding K, Ohberg L, Forssblad M, Fahlstrom M, Alfredson $\mathrm{H}$. Sclerosing injections to treat midportion Achilles tendinosis: a randomised controlled study evaluating two different concentrations of Polidocanol. Knee Surg Sports Traumatol Arthrosc. 2008;16(9):859-864.

18. Ohberg $\mathrm{L}$, Alfredson $\mathrm{H}$. Ultrasound guided sclerosis of neovessels in painful chronic Achilles tendinosis: pilot study of a new treatment. Br J Sports Med. 2002;36(3):173-175; discussion 176-177.

19. Hoksrud A, Ohberg L, Alfredson H, Bahr R. Ultrasound-guided sclerosis of neovessels in painful chronic patellar tendinopathy: a randomized controlled trial. Am J Sports Med. 2006; 34(11):1738-1746.

20. Hoksrud A, Bahr R. Ultrasound-guided sclerosing treatment in patients with patellar tendinopathy (jumper's knee). 44-month follow-up. Am J Sports Med. 2011;39(11):2377-2380.

21. Lind B, Ohberg L, Alfredson H. Sclerosing polidocanol injections in mid-portion Achilles tendinosis: remaining good clinical results and decreased tendon thickness at 2-year follow-up. Knee Surg Sports Traumatol Arthrosc. 2006;14(12):13271332. 
22. Zeisig E, Ohberg L, Alfredson H. Sclerosing polidocanol injections in chronic painful tennis elbow-promising results in a pilot study. Knee Surg Sports Traumatol Arthrosc. 2006;14 (11): 1218-1224.

23. van Sterkenburg MN, de Jonge MC, Sierevelt IN, van Dijk CN. Less promising results with sclerosing ethoxysclerol injections for midportion achilles tendinopathy: a retrospective study. Am J Sports Med. 2010;38(11):2226-2232.

24. Alfredson $\mathrm{H}$, Tol JL. Chronic pain in the achilles tendon. Aspetar Sports Medicine Journal. 2013.

25. Drotman. The Absorption, Distribution and Excretion of Alkylpolyethoxylates by Rats and Humans. Toxicology and Applied Pharmacology. 1980;52:38-44.

26. Padulo J, Oliva F, Frizziero A, Maffulli N. Muscles, Ligaments and Tendons Journal - Basic principles and recommendations in clinical and field Science Research: 2016 Update. Muscles Ligaments Tendons J. 2016;6(1):1-5.

27. Martinez-Silvestrini JA, Newcomer KL, Gay RE, Schaefer MP, Kortebein P, Arendt KW. Chronic lateral epicondylitis: comparative effectiveness of a home exercise program including stretching alone versus stretching supplemented with eccentric or concentric strengthening. J Hand Ther. 2005;18(4):411419, quiz 420.

28. Norregaard J, Larsen CC, Bieler T, Langberg H. Eccentric exercise in treatment of Achilles tendinopathy. Scand J Med Sci Sports. 2007;17(2):133-138.

29. Frizziero A, Trainito S, Oliva F, Nicoli Aldini N, Masiero S, Maffulli $N$. The role of eccentric exercise in sport injuries rehabilitation. Br Med Bull. 2014;110(1):47-75.

30. Lohrer $\mathrm{H}$, Nauck T. Cross-cultural adaptation and validation of the VISA-P questionnaire for German-speaking patients with patellar tendinopathy. J Orthop Sports Phys Ther. 2011; 41(3):180-190.

31. Lohrer $\mathrm{H}$, Nauck T. Cross-cultural adaptation and validation of the VISA-A questionnaire for German-speaking achilles tendinopathy patients. BMC Musculoskelet Disord. 2009; 10:134.

32. Germann G, Harth A, Wind G, Demir E. [Standardisation and validation of the German version 2.0 of the Disability of Arm, Shoulder,Hand (DASH) questionnaire]. Unfallchirurg. 2003; 106(1):13-19.

33. Iversen JV, Bartels EM, Langberg $\mathrm{H}$. The victorian institute of sports assessment - achilles questionnaire (visa-a) - a reliable tool for measuring achilles tendinopathy. Int J Sports Phys Ther. 2012;7(1):76-84.
34. Knobloch $\mathrm{K}$. The role of tendon microcirculation in Achilles and patellar tendinopathy. J Orthop Surg Res. 2008;3:18.

35. Zwerver J, Bredeweg SW, van den Akker-Scheek I. Prevalence of Jumper's knee among nonelite athletes from different sports: a cross-sectional survey. Am J Sports Med. 2011;39 (9):1984-1988.

36. Paavola M, Kannus P, Paakkala T, Pasanen M, Jarvinen M. Long-term prognosis of patients with achilles tendinopathy. An observational 8-year follow-up study. Am J Sports Med. 2000; 28(5):634-642.

37. Solveborn SA. "Tennis elbow" is usually caused by other than tennis. The earlier the treatment the better; spontaneous remission occurs often within 8-13 months. Lakartidningen. 1999;96(5):483-485.

38. Paoloni JA, Appleyard RC, Nelson J, Murrell GA. Topical glyceryl trinitrate treatment of chronic noninsertional achilles tendinopathy. A randomized, double-blind, placebo-controlled trial. J Bone Joint Surg Am. 2004;86-A(5):916-922.

39. Alfredson $\mathrm{H}$, Ohberg $\mathrm{L}$. Increased intratendinous vascularity in the early period after sclerosing injection treatment in Achilles tendinosis: a healing response? Knee Surg Sports Traumatol Arthrosc. 2006;14(4):399-401.

40. Leopold CS, Maibach HI. Effect of cutaneously applied nonionic surfactants and local anesthetic bases on thermal sensations. Pharmazie. 2004;59(1):50-54.

41. McCafferty DF, Woolfson AD, Boston V. In vivo assessment of percutaneous local anaesthetic preparations. $\mathrm{Br} \mathrm{J}$ Anaesth. 1989;62(1):17-21.

42. Alfredson $\mathrm{H}$, Ohberg L. Sclerosing injections to areas of neovascularisation reduce pain in chronic Achilles tendinopathy: a double-blind randomised controlled trial. Knee Surg Sports Traumatol Arthrosc. 2005;13(4):338-344.

43. Stasinopoulos D, Pantelis M, Stasinopoulos K. Comparing the effects of eccentric training with eccentric training and static stretching exercises in the treatment of patellar tendinopathy. A controlled clinical trial. Clin Rehabil. 2012;26(5):423-430.

44. de Vos RJ, Heijboer MP, Weinans H, Verhaar JA, van Schie JT. Tendon structure's lack of relation to clinical outcome after eccentric exercises in chronic midportion Achilles tendinopathy. J Sport Rehabil. 2012;21(1):34-43.

45. Docking SI, Ooi CC, Connell D. Tendinopathy: Is Imaging Telling Us the Entire Story? J Orthop Sports Phys Ther. 2015; 45(11):842-852.

46. Dirrichs T, Quack V, Gatz M, Tingart M, Kuhl CK, Schrading S. Shear Wave Elastography (SWE) for the Evaluation of $\mathrm{Pa}$ tients with Tendinopathies. Acad Radiol. 2016. 